gemäß zu arbeiten, d. h. die laufenden Arbeiten einzuteilen und somit ihre Arbeits. kräfte und Einrichtung voll auszunutzen, um so gerechter ist zugleich die Verteilung der Kosten. Der idealste Zustand wäre nach den bisherigen Erfahrungen wohl der, durchweg größere staatliche Nahrungsmittel-Untersuchungsanstalten einzurichten, und so die Unterhaltungskosten ganz gleichmäßig unter Berücksichtigung der gesanten Einnahmen des Staates an Geldstrafen und Untersuchungsgebühren aus $\S 16$ Abs. 4 des Nahrungsmittelgesetzes und den entsprechenden Bestimmungen der Ergänzungsgesetze zu diesem Gesetze an verteilen. Denn insbesondere in den leistungsschwachen kleinen Gemeinden sind die Kosten, die durch die ambulante Lebensmittel- sowie auch durch die hauptberufliche Weinkontrolle entstehen, verhältnismäß̊ig am größten. Es fragt sich demnach, ob eine allgemeine Verstaatlichung der öffentlichen Nahrungsmittel-Unterzuchungsanstalten möglich ist, und ob vielleicht demnächst sogar die Verhältnisse zu einer allgemeinen Verstaatlichung drängen werden. Die Beantwortung der ersten Frage hängt zum Teil mit der Leistungsfähigkeit und den Grundsätzen der Länder auf finanziellem Gebiet zusammen, jedoch darf bei der Prüfung dieser Frage uicht übersehen werden, daß der Staat durch die Festsetzung angemessener Gebühren die ihm durch die Verstaatlichung bestehender offentlicher Anstalten erwachsenden Kosten besser abzuwälzen in der Lage ist wie die Behörden, die die bisherigen öffentlichen Anstalten der zuvor angegebenen Art unterhalten, und dab gegebenenfalls dann diese Kosten sich für die Polizeibehörden voraussicbtlich wesentlich günstiger als bisher gestalten werden. Die Beantwortung der zweiten Frage ergibt sich aus der Entwickelung der finanziellen Lage der Gemeinden. Anscheinend ist diese bis auf weiteres im allgemeinen eine recht ungünstige. Ich möchte zur Zeit davon absehen, zu den beiden außerordentlich wichtigen Fragen weiter Stellung zu nehmen; der Zweck meiner Ausführungen sollte lediglich der sein, sie anzuschneiden, um hierdurch darauf hinzuweisen, daß auch diese Entwickelung der Verhältnisse sorgfältig weiter verfolgt werden $\mathrm{mub}$, und ich behalte mir vor, auf beide Fragen zurückzukommen, sobald sich das Ergebnis der gegenwärtigen Entwickelung der wirtschaftlichen Verhältnisse der Anstalten, der Gemeinden und des Staates klarer als bisher übersehen läbt.

Der Vorsitzende: Ihr Beifall ist das beste Zeugnis dafür, welehes lebhafte Interesse die Ausführungen des Berichterstatters gefunden haben. Auch ich möchte als Leiter der Versammlung dem Berichterstatter den aufrichtigsten Dank der Versammlung aussprechen.

Zunächst bitte ich Herrn Beythien, das Wort zu seinem Referat zu nehuen.

\title{
Ersatzmittelverordnung oder Nahrungsmittelgesetz?
}

\section{Von \\ A. Beythien-Dresden.}

De mortuis nil nisi bene! So ungefähr könnte man meines geschätzten Vorredners Ausführungen über das verflossene Ersatzmittelwesen charakterisieren, denen ich mich im großen und ganzen durchaus anschließe, deren Ergänzung durch scbärfere kritische Hervorhebung gewisser Einzelheiten mir aber doch nicht überflüssig erscheint. Wie Sie wissen, bin ich niemals ein Freund der Reglementierungs- und Organisationssucht der vergangenen Jahre gewesen, sondern mehrfach gegen die Flut von papierenen Verordnungen, die sich über unser Volk ergoß, aufgetreten. Sie 
werden es mir daher nicht verübeln, wenn ich beim Beginn einer newen Ära unserer Tätigkeit noch einmal die Schattenseiten der früheren Einrichtung hervorhebe, denn aus unseren Fehlern lernen wir.

Hervorgerufen durch die Not der Zeit und den daraus erwachsenden empörenden Schwindel mit Ersatzmitteln jeder Art, ist die Einfübrung des Genehmigungsz w anges in erster Linie, wir dürfen das nicht verschweigen, von den Vertretern der amtlichen Nahrungsmittelkontrolle gefordert und begrüßt worden. Sie hat jedoch die auf sie gesetzten Hoffnungen nur zu einem recht bescheidenen Teile erfüllt, dafür aber eine Reihe bedenklicher Übelstände gezeitigt, die eine Aufhebung der bezüglichen Vorschriften immer dringender wünschenswert erseheinen ließen.

Die Ursachen für das Versagen der mit bester Absicht errichteten Ersatzmittelstellen liegen jetzt klar vor aller Augen. Sie sind einerseits in dem ständig wachsenden Rohstoffmangel zu suchen, der die Herstellung brauchbarer, genehmigungswürdiger Ersatzmittel im Grunde überbaupt unmöglich machte, andererseits aber auch in dem bürokratischen Geschäftsgebaren vieler dieser Ämter und Stellen, deren Leitung nicht immer sachkundigen Persönlichkeiten anvertraut war. Auch ohne daß ich bestimmto Namen nenne, wissen Sie, daß manche Entscheidungen den Geist der Mittelmäßigkeit und subalterner Auffassung an der Stirn trugen.

Ein grundsätzlicher Febler war es schon, daß eine Reihe der wichtigsten Nahrungs- und Genufmittel dem Einflusse der Ersatzmittelstellen entzogen blieben, insbesondere nach der Verordnung vom 7. März 1918 1 ) alle Waren, deren Herstellung und Vertrieb von einer anderen dem Reichskanzler unterstehenden Stelle beaufsichtigt wurde, wie Kunsthonig und Vanillinzucker (Reichszuckerstelle), Pudding. pulver, Morgentrank und Würze, soweit das Rohmaterial durch die Kriegsnährmittel G. m. b. H. zur Verfügung gestellt wurde, Knochenbrühextrakt, Knocbenbrühwürfel und Knochenbrühwürfelmasse (Kriegsausschuß für pflanzliche und tierische Öle und Fette); Extrakte und Würzen aus Fischen, Schal- und Krustentieren, sowie Würste, Sülzen und sonstige Erzeugnisse aus diesen Stoffen, soweit sie nicht ohne weiteres als Konserven aus Fischen, Schalund. Krustentieren anzusehen waren (Reichskommissar für Fischversorgung); KaffeeErsatzmittel, soweit sie aus den vom Kriegsausschuß für Kaffee, Tee usw, gelieferten Rohstoffen hergestellt waren (Getreidekaffee, Zichorienkaffee, ferner KaffeeErsatzmischungen aus Getreide, Zichorie, Rüber, Eicheln, Obsttrestern, Eichelkaffee, Kaffee-Essenz aus Zuckerstoffen, Feigenkaffee); kün stliche (gestreckte) Marmeladen, Gelees und Muse (Kriegsgesellschaft für Obstkonserven und Marmeladen), weiter auch Margarine und Kunstspeisefett, Süßstoffe ${ }^{2}$ ), Bier, Tabak usw.

Mußte schon diese Verteilung auf zahlreiche Stellen, weil sie Zersplitterung und widerstreitende Auffassungen unabweisbar herbeiführte, als bedenklicher Fehler angesehen werden, so wirkte die Ausschaltung der in erster Linie berufenen Nahrungsmittelchemiker, oder doch ihrer überwiegenden Zahl, von den entscheidenden Stellen geradezu verhängnisvoll, weil sie das Interesse der letzteren lähmte und die praktische Durchführung der gefaßten Beschlüsse ungünstig beeinflußte.

Zweifellos sind zunächst die allerschlimmsten Auswüchse des Ersatzmittelschwindels, die Kreidepulver und ähnliche Scheußlichkeiten beseitigt worden, aber die tatsächlich zum Handel zugelassenen Erzeugnisse waren doch zum gröBten Teile nicht viel besser als ihre abgelehnten Genossen. Ich will den Ersatzmittelstellen aus ihrer oft recht weitherzigen Stellung bei Genehmigungen keinen allzu großen Vorwurf machen, habe ich doch selbst empfohlen, das Publikum durch Suggestion und harmlosen Selbstbetrug zu beruhigen, statt ihm die gähnende Leere der Nahrungsmittel-

1) Vergl. Juckenack, Diese Zeitschrift 1919, 37, 213.

2) Mitteil. f. Preispritiungsstellen vom 1. Oktober 1919. N. $21_{x}$ 
geschäfte zu zeigen. Ich will auch, um nicht alte Wunden aufzureißen, lieber die Toten ruhen lassen und nicht einzelne genehmigte Ersatzmittel, die wie Suppenmehl, Puddingpulver, Vanillinsalz und Honigaroma einigen unter uns ans Herz gewachsen sind, näher beleuchten. Aber darin stimmen wir doch wohl überein, daß mit genehmigten Ersatamitteln manche wertvolle Rohstoffe, besonders Mehl und Zueker, in überflüssigen und unzweckmäßigen Mischungen mit Rübe und Salz vergeudet wurden, die besser den Verbrauchern unvermischt zugeführt worden wären.

Vor allem aber bot der für $50 \mathrm{M}$. ausgestellte Genehmigungsschein, und das ist die bedenklichste Seite der ganzen Angelegenheit, nicht die mindeste Gewähr dafür, daf die unter seiner Deckung in den Handel gebrachte Ware nun auch wirklich der dem Ersatzmittelamte eingereichten und von diesem mit heißem. Bemühen untersuchten Probe entsprach. Wir Vertreter der amtlichen Nahrungsmittelkontrolle wissen im Gegenteil nur zu gut, daß das durchaus nicht der Fall war. Während der ehrliche Gewerbetreibende sich vielfach scheute, seine langjährig erprobten Rezepte einem ihm gänzlich unbekannten Chemiker preiszugeben, machte der gewerbsmäßige Schwindler einfach eine wundervolle Untersuchungsprobe ad usum delphini zurecht und füllte dann in die mit dem Genehmigungsvermerk versehene Umbüllung - die letztere war ja eigentlich die Hauptsache - eine beliebige andere wertlose Mischung. Noch vor wenigen Tagen ist mir bekannt geworden, daß ein Waschmittelfabrikant aus der Dresdner Gegend, nachdem ihm die früher erteilte Genehmigung wieder entzogen worden war, für $500000 \mathrm{M}$. Kochsalz als Waschpulver an den Mann brachte, und jeder von Ihnen hat ähnliche Fälle erlebt.

Was machte nun der praktische Nahrungsmittelchemiker, dem grofmütig die Überwachung des Handels überlassen war, wenn er ein ihm wertlos erscheinendes Ersatzmittel antraf? Er sah zunächst, wenn er besonders gewissenhaft und organisatorisch veranlagt war, in seiner an der Hand der veröffentlichten Listen angefertigten Karthotek nach und konnte so ohne weiteres feststellen, ob ein genehmigtes oder abgelehntes Ersatzmittel in Frage kam.

Aber mit diesen Karteien hatte es seinen Haken. Sie wuchsen und wuchsen und schwollen bald so unendlich an, daß sie alle Räume des Untersuchungsamtes äberfluteten und einen ganzen Stab von Kanzleibeamten zur Bedienung erforderten. Und schließlich nützten sie doch nicht weiter, als daß sie die Möglichkeit einer An. frage bei der zuständigen Ersatzmittelstelle gewährten, ob sie in Wahrheit die Genehmigung erteilt habe. Bei einer Abweichung von den Richtlinien ersëhien die Verneinung dieser Frage aber selbstverständlich! So war nicht nur die vorherige Untersuchung der eingelieferten Probe eine Verschwendung geistiger Energie, da ja die Genehmigung schon auf Grund des bekanntgegebenen Rezeptes erteilt werden konnte, sondern auch die ganze Arbeit der Ersatzmittelstelle war überflüssig, weil der Schwerpunkt der Überwachung bei der späteren Kontrolle lag, deren Wirksamkeit durch sie nur behindert wurde. Völlig zwecklos mußte sie aber mit dem Aufhören des Kriegszustandes werden, als alle diese schönen genehmigten Ersatzmittel mit einem Schlage unverkäuflich wurden und sich in Fabriken und Läden als toter Ballast anhäuften.

Wenn sonach heute die geplagten Kleinbändler die Authebung des Genehmigungszwanges freudig begrüßen, so wird ihm auch, abgesehen von den unmittelbar an den Ersatzmittelstellen beschäftigten, kaum ein Nahrungsmittelchemiker eine Träne nachweineu. Fr war ein Notbehelf, und noch dazu ein untauglicher, im Kriege und mußte trotz allen Widerstrebens wie die übrigen Errungenschaften der Zwangswirtschaft weichen.

$J_{n}$, nach meiner Ansicht wäre es am besten gewesen, um einmal reine Bahn zu schaffen, dem Genehmigungšwang gleichzeitig sämtliche anderen auf Nahrungs- 
und Genußmittel bezügliche Kriegsverordnungen, mit alleiniger Ausnahme der Bekanntmachung gegen irreführende Bezeichnungen vom 26. Juni 1916, nachzuschicken, denn sie verwirren nur die Begutachtung, ohne einen praktischen Nuizen zu stiften.

So sollte, um nur ein Beispiel herauszugreifen, die Bekanntmachung des Bundesrats über die äufere Kennzeichnung der Waren vom 18. Mai 1916 das Publikum zur Mitwirkung bei der Bekämpfung des Nahrungsmittelschwindels heranziehen, indem man ibm die Möglichkeit verschaffte, aus dem Aufdruck Inhalt und Preis zu ersehen. Man überließ den Käufern die Entscheidung, ob es die geforderten Preise bezahlen wolle, nachdem man die meist unverschämte Forderung selbst nicht hatte verhindern können, auch nicht mit Hilfe der noch kurz zu besprechenden Bekanntmachung gegen äbermäßige Preissteigerung. Aber es zeigte sich, daßs das Publikum, dessen Urteilslosigkeit schon im Frieden oft beklagt werden mußte, sich in den Tahren der Not gar nicht um die schönen Inschriften der Verpackungen kümmerte. Was nützte ihm die Kenntnis, då der ihm für $75 \mathrm{Pfg}$. verabfolgte Papierbeutel 2 oder $3 \mathrm{~g}$ Pfeffer enthielt, oder daß die $10 \mathrm{M}$ : kostende Blechdose 3 Heringe mittlerer Größe umschloß! Er gebrauchte eben den Pfeffer und bezablte daher den 60-fachen Friedenspreis, und wenn der Verkäufer statt 75 Pfg. 1,50 M. auf den Beutel gedruckt hätte, so würde die Hausfrau eben den 100 -fachen Betrag des Friedenspreises bezahlt haben. Die Bekanntmachung verschaffte somit zwar den. Behörden das angenehme beruhigende Gefühl, daß eine Täuschung der Käufer unmöglich gemacht sei, aber das war auch alles! Die Zwischenhändler verteilten nach wie vor die Gewürze auf Papierbeutel mit $2-3 \mathrm{~g}$ Inhalt und schrieben gewissenhaft ihren Namen und Wohnort, das Gewicht und den Preis der Ware auf die Umhüllung. Gekauft wurde doch! Wenn wirklich einmal ein eifriger Nahrungsmittelchemiker feststellte, daß statt der zugegicherten $3 \mathrm{~g}$ nur $2^{1 / 2} \mathrm{~g}$ Pfeffer in der Packung ent. balten waren, so wurde ihm bewiesen, daß die Ware inzwischen durch Eintrocknung um $1 / 2 \mathrm{~g}$ leichter geworden sei, oder daß nur ein unvermeidliches Versehen der Arbeiter vorliege. Jeder in der amtlichen Kontrolle einigermaßen Erfahrene hätte den Vätern dieser Verordnung sagen können, daß die Hoffnung auf Mitwirkung der Kaufer illusorisch sei, daß das, Publikum, wenn überhaupt, gegen seinen Willen vor Betrug geschützt werden müsse.

Insofern lag der Bekanntmachung gegen abbermäßige Preissteigerung vom 23. Juli 1915 ein richtiger Gedanke zugrunde, und es soll nicht bestritten werden, daß durch die aufopfernde Arbeit der Preisprüfungstellen, Landespreisprüfungsund Kriegswucherämter mancher Schwindel aufgedeckt und geabndet worden ist. Aber im großen und ganzen kann man sich doch dem Eindruck nicht éntziehen, daß mehr die kleineren plumperen Betrügereien erwischt wurden, während die großèn Millionenschwindler durch die Maschen des Gesetzes schlüpften.

Das lag, abgesehen von der Schwierigkeit, den Geltungsbereich der Bekanntmachung genau abzugrenzen, - hat man doch sogar gezweifeli; ob Rum und Feigen Gegenstände des täglichen Bedarfs seien, - vor allem an der Zaghaftigkeit, mit der man an die Feststellung eines übermäßigen Gewinns herantrat.

Zwar machten es sich einige Chemiker, die nach dem Vorbilde Jonscher's ${ }^{1}$ ) frisch, fromm, fröhlich und frei an die Sache herantraten, insofern leicht, als sie den für eine bestimmte Warenmenge geforderten Preis mit dem im reellen Handel üblichen Marktpreise größerer Gewichtseinheiten (1 Pfund, 1 Kilogramm) verglichen und bei offensichtlicher Überschreitung des letzteren einen übermäßigen Gewinn annahmen. Aber dieses vereinfachte Verfahren hielt, wie schon Stadlinger ${ }^{2}$ ) hervorhob, einer

1) Zeitsehr. öffentl. Chem, 1918, 24, 268, 285; 1919, 95, 148,

7) Ebenda 1919, 25, 275 . 
kaufmännischen Kritik gegenüber nicht stand. Einige Beispiele aus Jonscher's Sammlung mögen das näher illustrieren (wörtlich):

"Ein Blechschächtelchen mit 86 Tabletten von insgesamt $38,5 \mathrm{~g}$ Gewicht Te wurde zu 1,50 M. verkauft. Der Verkaufspreis des Tees berechnete sich nach Abzug des Verpacknngswertes mit $10 \mathrm{Pfg}$. leieht $\mathrm{zu} 18,18 \mathrm{M}$. pro Pfund, womit eine 11 -fache Wertäberforderung vorlag, wenn man das Pfund Teegrus mit 1,50 M. reellem Werte einsetzte.

Über einen zu $20 \mathrm{Pfg}$. verkauften Buttersparer, der aus $20 \mathrm{~g}$ schwach gesalzenem und gefärbtem Kartoffelmehl bestand, urteilt Jouscher: ,Da der Beutelinhalt genau $50 \mathrm{~g}$ wog und mit $20 \mathrm{Pfg}$. verkauft wurde, so kam das Pfund Buttersparer, dessen reeller Handelswert unter Zagrundelegung des gesetzlichen Höchstpreises für Kartoffelmelal sich nur auf 19,8 Pfg. berechnete, auf 2 M. pro Pfund zu stehen. Der verlangte Kleinverkanfspreis äberstieg also den reellen Handelswert um das 10-fache, was ibm eine Beanstandung wegen Preisüberforderung eintrug."

Wie ersichtlich litt diese Überlegung, ganz abgesehen von den viel zu niedrigen Marktpreisen, zu denen die Rohstoffe gar nicht erlangt werden konnten, an dem grundsätzlichen Fehler, daß sie Übervorteilung des Käufers mit übermäßigem Gewinn des Herstellers verwechselte. Zum Nachweise des letzteren war vielmehr Berücksichtigung der Geschäftsunkosten erforderlich, und man muß Stadlinger (a. a. O.) recht geben, wenn er sagt:

Dab́ der Hersteller doch sehr beträchtlichen Aufwand für das Mischen der Rohstoffe, für Abfüllen, Mischverlust, Umbüllung, Kisten, Frachten, Rollgeld und sonstige Be riebskosten zu bestreiten hat, daf er schließlich auch Anspruch auf angemessene Verzinsung des eigenen und des fremden Betriebskapitals, anf den Ausgleich von Verlusten, sowie Entsclädigung für seine eigene Arbeitskraft erheben muk, schien den betreffenden Gutachtern bei der Kalkulation des Selbstkostenpreises keiner weiteren Beachtung wert."

Auch wenn man weniger sanguinisch wie Stadlinger über den volkswirtschaftlichen Wert dieses meist ganz überflüssigen Mischens, Abfüllens in kleine Päckenen.u. dergl. denkt, so ist doch nicht zu bestreiten, daß die Gerichte diese Kosten bei Erörterung der Schuldfrage berücksichtigen muften. Wieweit sie in dieser Richtung gingen, mögen einige Beispiele aus meiner eigenen Praxis dartun:

Fin Winkelfabrikant füllte für $3 \mathrm{Pfg}$. gelbgefärbtes Kartoffelmehl in einen Papierbeutel, der mit der Bezeichnung Butterpulver für $20 \mathrm{Pfg}$. im Kleinhandel abgegeben wurde. Der Kăufer war zwreifellos um 17 Pfg. geschädigt, aber eine übermäßige Preissteigerung wurde vom Gerichte verneint, weil der Hersteller folgende, von dem praktischen Sachverständigen der Preisprüfungsstelle gebilligte Kalkulation vorlegte:

Wert des Kartoffelmehls . . . . 3 Pf. Z Zeitungsreklame . . . . . . . 2 Pfg.

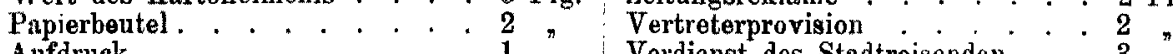

Aufdruek . Verdienst des Stadtreisenden : : 3 "

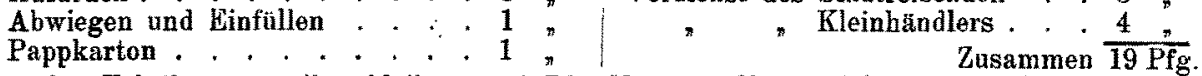

Für den Fabrikanten selbst bleibt nar 1 Pfg. Natzen. Niemand hat einen übermäßigen Gewinn, aber das Publikum ist der Dnmme.

Ein weiteres Beispiel für technische Waren, das ich bereits auf unserer 16. Hauptversammlung in Berlin am 28. September 1918') vorzutragen beabsichtigte, erseheint fast noch lehrreicher und möge daher hier angeführt werden Ein Dresàner Fabrikant brachte im Jahre $1916 \mathrm{M}$ is chso d a aus $20 \%$ Soda und $80 \%$ Steinsalz zum Preise von $9 \mathrm{M}$. für $100 \mathrm{~kg}$ in den Handel. Diesen Preis begrändete er im Laufe der Voruntersuchung wegen iubermäßiger Preissteigerung in folgender Weise:

$20 \mathrm{~kg}$ calc. Soda $(100 \mathrm{~kg}=11,50 \mathrm{M})$. . . . . . . 2,30 M.

$80 \mathrm{~kg}$ Steinsalz $(100 \mathrm{~kg}=1,58 \mathrm{M}$.) . . . . . . . . . 1,27 "

Betriebsunkosten, wie Ärbeitslohn, Miete . . . . . . . 1,50"

Sack für die fertige Mischung . . . . * . . . . . . . 1,70 ,

Abnutzung der Säcke für Rohstotfe. . . . . . . . . 0,70 ,

Vergallungsspesen . . . . . . . . . . . . . . $0.47^{*}$

Summe $7,94 \mathrm{M}$.

Reingewinn 1,06 ,

1) Diese Zeitschrift 1919, 37, 353. 
Gegen diese Berechnung waren von der Preisprüfungsstelle keine Einwendungen erhoben worden, bis ich darauf hinwies, daf der relativ nicht besonders hohe Betrag für Reingewinn und Betriebsunkosten doch ein ganz anderes Gesicht orhalte, wenn man den Umfang des ganzen Geschäftes berücksichtige. Der sog. Fabrikant hatte nämlich in dem kurzen Zeitraum von 22 Monaten $685000 \mathrm{~kg}$ Soda bezogen, aus denen unter Zusatz der vierfachen Menge Salz $34250 \mathrm{dz}$ Mischsoda hergestellt worden waren. Als Betriebsunkosten berechnete er für $1 \mathrm{dz}$ 1,50 M., im ganzen also mehr als 51000 M. Diese Betriebsunkosten entstanden dadurch, dak die Ebefrau des Unternebmers mit 2, zeitweilig sogar 3 Arbeitern die Soda und das Salz in eine Mischtrommel schütteten und nach mehrmaliger Umdrehung der Kurbel mit Handantrieb das Gemisch in Säcke füllten. Rechnet man die Löhne der Arbeiter für damalige Zeit hoch zu $7000 \mathrm{M}$., so erhielt die Frau für ihre gewißs nicht übermäßjig geistvolle Tätigkeit in 22 Monaten $44000 \mathrm{M}$. Dazu der sog. Reingewinn von $1,06 \mathrm{M}$. für $100 \mathrm{~kg}$, oder rund $36000 \mathrm{M}$. in 22 Monaten ergibt als die Gesamteinnahme des Ehepaares für die Beglüekung der Menschheit mit versalzener (sog. "Dresdner") Soda den Betrag von 80000 M.! Diese Überlegung, die ja nicht eigentlich Sache des Chemikers war, fand beim Gericht Verständnis und hatte den vom Standpunkte des moralischen Gefühls erfreulich ${ }^{2}$ Erfolg, daß̧ dem "Sodafabrikanten* ein Teil des Ruubes wieder abgejagt werden konnte. Das ändert aber nichts an der Tatsache, dak in zahllosen anderen Fallen gewagte Aufstellangen von Geschäftsunkosten die kritische Untersuchung der Preisprüfungsstellen bestanden.

Ist man doch in der Anrechnung sog. Spesen soweit gegangen, daß ein Eraatzfabrikant einen Rucksack, den er zum Einhamstern von Butter gebrauchte, mit 5 M. und Duden's Rechtschreibung zur Vermeidung orthographischer Fehler beim Rechnungschreiben mit 3,60 M. einsetzen durfte. Es fehlte nur noch, daf man die Kontoreinrichtung, ein Fahrrad und einen Sportanzug auf den Preis draufschlug. So gehandhabt, mubte sich die Bekanntmachung gegen übermäßige Preissteigerung als eine stumpfe Waffe erweisen. Das einzige Mittel, sie wirksam zu gestalten, nämlich den Grundsatz, daß die Spesen einen bestimmten Betrag des Materialpreises, etwa das Doppelte, nicht übersteigen durften, hat man nicht zu ergreifen gewagt.

Gerade weil man die Wirkungslosigkeit dieser beiden Verordnungen zur Bekämpfung des Ersatzmittelschwindels erkannte, hat man den Genehmigungszwang eingeführt. Nachdem also nunmehr der letztere, als die ultima ratio gefallen ist, sollte man meiner Ansicht nach auch ihre kümmerlichen Vorgänger preisgeben, denn wenn der Herzog fällt, muf der Mantel nach.

Bis das geschieht, wollen wir uns wenigstens freuen, daß das Nahrungsmittelgesetz wieder in seine vollen Rechte tritt, und nur darum habe ich die Ihnen vielleicht abseits vom Thema liegende Kritik der Kriegsverordnungen etwas ausführlicher gestaltet, um die Vorzüge des früheren gediegenen, wohldurchdachten Reichsgesetzes in das richtige Licht zu setzen.

Die uns altvertraute Waffe zur Bekämpfung der Unlauterkeit im Nahrungsmittelhandel, das Nahrungsmittelgesetz vom 14. Mai 1879, das für alle übrigen Staaten vorbildlich gewesen ist, wird sich auch in den kommenden Zeiten wieder bewähren, und wenn wir auch schon seit Jahren über Vorschläge zu seiner Ergänzung und Verbesserung beraten haben, so möchte ich es doch in seinen Grundzügen erhalten wissen, schon aus dem Grunde, weil es in einer Unzahl wertvoller richterlicher Urteile bis in die feinsten Einzelheiten klargestellt worden ist, und sein Inhalt tief in das Rechtsbewußtsein des Volkes Eingang gefunden hat.

Vor allem würde es mir bedenklich erscheinen, die freie richterliche Beweisführung zu sehr einzuschränken, nicht nur vom Standpunkte des Beschuldigten aus, der sich dadurch beeinträchtigt füblen müßte, sondern auch in unserem eigenstèn Interesse. Denn ohne die Verdienste des geschickten Analytikers irgendwie zu unterschätzen und bei voller Würdigung der Kämpfe von Gegensachverständigen mit ihren unangenehmen Begleiterscheinungen balte ich doch die sorgfältige sachliche Begründung unserer Gutachten für den reizvollsten Teil unserer Tätigkeit.

$\mathrm{Da} \beta$ das Nahrungsmittelgesetz auch bei Berücksichtigung dieser allgemeinen Gesichtspunkte eine Reihe von Mängeln und Lücken enthält, die in später erlassenen 
Gesetzen anderer Staaten, besonders der Schweiz, beseitigt worden sind, ist uns allen bekannt und wir werden uns nicht der Aufgabe entziehen, unsere praktischen Erfahrungen bei einer Neuregelung zur Verfügung zu stellen.

Im Hinblick auf die eingehenden Darlegungen meines Vorredners, den wir als einen der besten Kenner dieser Frage kennen, darf ich mich darauf beschränken, die dringendsten Punkte herauszugreifen, um so mehr, als ich in großen und ganzen mit ihm völlig übereinstimme.

Ûbersehrift des Gesetzes. Fraglich ist mir zunächst nur, ob man in der Uberschrift des Gesetzes, wie Herr Geheimrat Juckenack empfiehlt, die Ausdrücke Nahrungs- und Genußmittel in den Begriff "Lebensmittel" zusammenfassen soll. Mir scheint das letzlere Wort weniger bezeichnend, denn wenn es bislang auch in der Regel für die zur menschlichen Ernährung dienenden Stoffe angewandt worden ist, so könnte es doch rein sprachlich auch auf andere wichtige Gegenstände des täglichen Bedarfs, wie Heiz-, Leucht- und Kleidungsstoffe ausgedehnt, oder gar; was bedenklicher wäre, mittels gewiegler Dialektik auf die zur Ernährung n o tw endigen Stoffe eingeschränkt werden. Gerade das von Hern Geheimrat Juckenack angezogene Urteil des Reichsgerichts vom 27. November 1917, nach welchem Tabak und Zigaretten nicht unter den Begriff Lebensmittel fallen, redet in dieser Hinsicht eine bedenkliche Sprache.

Ich glaube, wenn die altgewohnten Ausdrücke: Nahrungs- und Genufmittel beibehalten werden, können wir am ersten erwarten, daß die Rechtsprechuug mit dem Wiederèintritt geordneter Verbältnisse zu der früher vom Reichsgericht gegebenen Begriffsbestimmung zurückkebren wird, nach der es heißt:

Nahrungsmittel sind Stoffe, welche, sei es in fester oder flussiger Form, der $\mathrm{E}$ nährung des menschlichen Körpers dienen, auch wenn zu deren Genjefbarkeit eine vorherige Zubereitung erforderlich ist.

Der Begriff Genufmittel ist weiter. Er umfafist alle Stoffe, welche auch ohne der Ernährung (d. h. der Erzeugung von Kraft und Wärme oder dem Ersatze verbrauchter Körpersubstanz. B.) zu dienen, genossen zu werden pflegen, allerdings unter der Voraussetzung, dafs sie durch die Organe dem menschlichen Körper zugefuhrt und mit dem Genusse verbraucht werden.

In diesem Sinne sind labak und Zigarren in dem Urteil des Reichsgerichts vom 31. März 1881 ${ }^{1}$ ) als Genußmittel angesehen worden, womit den Forderungen der Nahrungsmittelkontrolle völlig Genüge geleistet sein würde.

Im übrigen würde es keine Schwierigkeiten bieten, die Begriffsbestimmung für Nahrungs- und Genußmittel in das Gesetz mit aufzunehmen.

Zustimmen wird man Jucken ack in der Forderung, daß dic Vorschriften über Gebrauchsgegenstände gründlich nou bearbeito werden müssen und da6 statt ihrer in der Uberschrift des Geaetzes besser , andere Gegenstände des täglichen Bedarfs“ gesagt wird.

Ausdehnung der Kontrolle auf Fabrik- und Lagerräume. Der wiohtigste Punkt ist zweifellos die Erweiterung der den Polizeibeamten in $\$ 2$ N.M.G. eingeräumten Befugnisse. Mit Recht beschweren sich die Kleinhändler darüber, daß sie fast ausschließlich die Last der Kontrolle zu tragen haben und daß die Beschränkung der Revisionen auf die Ladengeachäfte eine außerordentliche Härte darstellt. Es ist. daher anzustreben, daß den Beamten und auch den Sachvorsuändigen der Polizei das Recht verliehen wird, in die Räumlichkeiten einzutreten, die zur Herstellung; Gewinnung, Behandlung und Aufbewahrung der in $\$ 2$ genannten Waren dienen. Sicher würde es sich auch nach den Muster des Schweizerischen Nahrungsmittelgesetzes empfehlen, den Beamten neben dem Rechte des Eintritts und der Probenahme auch das Recht der Besichtigung und der Ausführung einfacher Vorprüfungen zu erteilen.

1) Rechtspr. $8,848$. 
Auf diese Weise könnte der Schwerpunkt der Kontrolle in die Fabriken verlegt und die Verfälschung an der Wurzel bekämpft werden.

Den Vorschlägen Juckenack's zu den $\$ \S 3$ und 4 stimme ich vorbehaltlos zu. Die Auregung, den Milchbandel konzessionspflichtig zu machen, entspricht einem alten Wunsche der reellen Gewerbetreibenden und wird daher kaum auf besondere Schwierigkeiten stoßen. Ob die Ausdehnung der Vorschriften des N.M.G. auf die Konsum vereine ebenso glatt verlaufen wird, erscheint zunächst zweifelhaft. Wir müssen aber zum mindesten diese Forderung vertreten, da wir aus zahlreichen Beschwerden des Publikums wissen, daß auch diese Stellen nicht vom rein humanitären Standpunkte, sondern meist nach rein kaufmännischen Grundsätzen geleitet werden, so daß auch ihre Käufer sich of durch die verabfolgten Waren geschädigt fühlen. Mir ist die Rechtsprechung, daß in den Konsumvereinen kein Feilhalten oder Verkaufen stattifindet, bei dem klaren Wortaute des Gesetzes immer unverständlich gewesen. Da diese merkwürdige Auslegung nun aber einmal ergangen ist, müßte sie für die Zukunft durch eine besoudere Bestimnung des Gesetzes verhindert werden.

Die in $\S \tilde{5}$ erteilte Ermächtigung, durch kaiserliche Verordnung in bestimmten Fällen bindende Vorschriften zu erlassen, ist an die Voraussetzung "zum Schutze der Gesundbeit" gebunden. Alle vorliegenden Erfahrungen zwingen zu der Erweiterung auf den wirtschaflichen Schutz der Verbraucher.

Auch erscheint es mir zweckmäßig, um die Sondergesetze über Farben und bleizinkhaltige Gegenstände durch Ausführungsbestimmungen im Rahmen des allgemeinen Nahrungsmittelgesetzes entbehrlich zu machen, die in Absatz 4 aufgezăhlten Gruppen vou Gebrauchsgegensıảnden: Bekleidungsgegenstände, Spielwaren, Tapeten, EB-, Trinkund Kochgeschirr entsprechend zu erweitern.

So hat es sich, um nur einige Beispiele harauszagreifen, als gtorend herausgestellt, dabs Fafhähne, Senfbüchsen, Stanniolkapseln, Konservendosen usw. nicht als Eहs', Tink- und Kochgeschirre angesehen 'wurden Man könnte daher den letzteren nach dem Muster der Schweizerischen Verordnung vom 8. Mai 1914 ${ }^{1}$ ) an die Seite stellen : , bei der Zubereitung oder beim Genuf von Lebensmitteln zur Verwendung gelangende Werkzeuge und Einrichtungen und zur Aufbewahrung vou Lebensmitteln bèstimmte Gefäke" und, wie ieh hinzusetzen möchte, Umbüllungen.

Von anderen Gebrauchsgegenständen, bei denen die Verwendung schädlicher Stoffe gesundheitliche Störungen herbeiführen kann, wären hier noch Farbstifte und Malerfarben, Buntpapier, Kosmetika, Kerzen u. a. namhaft zu machen.

Die $\$ \S 6-9$ sind von Herrn Geheimrat Juckenack so erschöpfend und ganz in Übereinstimmung mit meiner Auffassung besprochen worden, daf ich mir ein näheres Eingehen ersparen kanu.

Dafür möchte ich aber dem $\$ 10$, dem eigentlichen Rückgrat unserer amtlichen Tätigkeit noch ein paar Worte widmen.

Als Hindernis bei der Verfolgung objektiver Beanstandungen hat sich oft das Fehlen einer Definition für die Begriffe ,verfälscht, nachgemacht, verdorben, gesundheitsschädlich" herausgestellt. Ist es mir doch passiert, då ein Gegensachverständiger die Verdünnung von Himbeersaft mit Wasser statt als Verfälschung als Verbesserung bezeichnete, weil der sanere Geschmack dadurch gemildert werde!

Es läge nun nahe, und diesen Vorsehlag habe ich früher in meinem Handbuche der Nahrungsmitteluntersuchung gemacht (Bd. IV, S. 51), die entsprechenden Begriffs. bestimmungen nach der seitherigen Rechtsprechung in das Gesetz mit aufzunehmen, Nach der jetzigen Sachlage erscheint es mir jedoch zweckmäßiger, diese Definitionen in die Ausführungsbestimmungen zu verweisen, dafür aber den Geltungsbereich der $\S \S 10-14$ auf ,irreführend bezeichnete" Nahrungs- und Genußmittel auszudebnen. Nur hierdurch läßt sich der mebrfach empfundene Ủbelstand beseitigen, daß gegen 254,263 .

1) Gesetze und Verordnungen, betr. Nahrungs- und Genufmittel, 1914, 6, 352, Artikel 66, 
den Verkauf von Pferdefleisch für Rindfleisch, von Rüböl für Olivenöl mit dem Nahrungsmittelgesetz nicht eingeschritten werden kann.

Als weitere Lücke des $\$ 10$ erwähne ich noch, dafo gegen die Feilhaltung verfälschter usw. Nahrungsmittel nur dann vorgegangen werden kann, wenn sie unter einer zur Täuschung geeigneten Bezeichnung erfolgt, nicht aber, wenn gar keine Bezeichnung vorhanden ist. Die Aufnahme einer Vorschrift, daß jedes verfälschte, nachgemachte oder verdorbene Nahrungsmittel die entsprechende wahrheitsgemäße Bezeichnung tragen muß, scheint mir unerläßlich.

Erlassen sie es mir, auf die übrigen Vorschriften des Nahruugsmittelgeselzes, die zweifellos ebenfalls von höchster Bedeutung, aber mehr verwaltungsrechtlicher Natur sind, näher einzugehen. Ich babe in dieser Hinsicht den Ausführungen meines Freundes Juckenack nichts hinzuzusetzen. Wohl aber möchte ich noch einmal auf die ungeheure Wichtigkeit der auf Grund von $\$ 5$ zu erlassenden Ausführungsbestimmungen, die ich mir als rechtsverbindliche Festsetzungen fiir die einzelnen Nahrungsmittel denke, hinweisen.

Wie ich schon mehrfach, zuletzt in meinem Vortrag auf der 13. Hauptversanmmlung. in Koblenz am 23. Mai 1914') ausgeführt habe, richtete sich der Widerstand der Gewerbetreibenden gegen die Nahrungsmittelkontrolle, vor allem gegen die sog. R e ch tsunsicherheit, die darin besteht, daß die gleiche Ware nicht von allen Gerichten in gleicher Weise beurteilt wird. Die Ursache dieses zweifellos vorhandenen, wenn auch oft übertriebenen Übelstandes liegt darin, daß die einzelnen Sachverständigen über den Begriff der normalen Beschaffenheit verschiedene Ansichten haben können. Wennschon es sich hier weniger um einen besonderen Mangel der Nahrungsmittel. kontrolle als um eine allgemeine Folge der Sachverständigen-Vernehmung handelt, die in gleicher Weise bei der Rechtsprechung über den Handel mit Webwaren, Mineralien zutage tritt, so ist doch die Beseitigung oder zum mindesten die Einschränkung derartiger: Widersprüche dringend erwünscht. Nachdem weder die sog. „Vereinbarungen", noch das vom Bunde der Nahrungsmittelfabrikanten herausgegebene "Deutsche Nahrungsmittelbuch" bei den Vertretern der Fabrikanten und Händler einerseits und der Nahrungsmittelkontrolle andererseits völlige Zustimmung gefunden haben, scheint der Erlab rechtsverbindlicher Festsetzungen über die Beschaffenheit der einzelnen Nahrungsmittel, Genufmittel und Gebrauchsgegenstände als einziges Mittel zur Herbeiführung gleichmäßiger Rechtsprechung allseitig angesehen $\mathrm{zu}$ werden. Wie bekannt, hat das Reichsgesundheitsamt unter Zuziehung von Sachverständigen aus den Handels- und Gewerbekreisen, sowie von praktischen Nahrungsmittelchemikern in mühsamer Arbeit die Abschnitte „Speisefette“, „Honig", „Essig“, „Käse“, „Kaffee“ und „Kaffeeersatzstoffe" fertiggestellt, und es steht zu hoffen, daß weitere Gegenstände alsbald in Angriff genommen werden, damit sie nach Neubearbeitung des Nahrungsmittelgesetzes alsbald ebenfalls in Kraft treten können. Wir dürfen uns rühmen, wertvolle Vorarbeiten über die Beurteilung von Wurstwaren, Milch, Speisefetten und -ölen, Marmeladen, alkoholfreien Getränken, Dessertwein, Trinkbranntwein, Gewürzen, Kakao und Schokolade geleistet zu haben, und werden uns auch in Zukunft der Verpflichtung nicht entziehen, die wissenschaftlichen Grundlagen für die erwünschten Verordnungen zu liefern. Wichtige Gebiete unseres Tätigkeitsbereichs warten ja noch der Regelung. Ich erinnere nur an Fleisch, Getreide, Mehl und Brot, Teigwaren, Bier und besonders die schwierige Materie der Gebrauchsgegenstände. Ihre alsbaldige Inangriffnabme scheint mir die dringendste Aufgabe des Vereins in den nächsten Jahren. Gediegene wissenschaftliche Leistungen werden nicht nur in idealem Sinne jedem einzelnen von uns die schönste Befriedigung verschaffen, sondern auch am wirksamsten das Ansehen der Vereins und unsere wirtschaftlichen und Standesinteressen fördern.

1) Diese Zeitschrift $1914,28,575$. 
Der Vorsitzende: Ioh danke dem Berichterstatter für die uns gemachten Ausführungen und möchte nun bitten, in die Diskussion über das erste Referat cinzutreten.

\section{Diskusion.}

Dr. Stadlinger: Der Vorredner hat bereits darauf hingewiesen, dal man über die 'Toten nichts Ungünstiges sagen soll. Ich für meine Person möchte etwas Günstiges sagen. Ich bedanere, daf die Frsatzmittelverordnung so sang. und klanglos gefallen ist, obne daf man etwas Besseres für die nächste Zeit an deren Stelle setzen konnte. Meines Ernchtens hätte das Nahrungsmittelgesetz so weit vorbereitet sein müssen, dak es automatisch an die Stelle der in Grund und Boden gewirtschafteten Ersatzmittelverordnung sofort eingetreten wäre. Wir haben jetzt ein Vakuum für die nächste Zeit, das auszufüllen große Sohwierigkeiten haben wird. Die Lrsatzmittelverordnung hat zweifellos ihr Gutes gehabt. Die Industrie hat gewuft, woran sie war, und ich bin darchaus nicht der Optimist, der glaubt, jetzt hören alle Ersatzmittel von selbst anf. Ich bin im Gegenteil der Meinung, dafs der Schwindel jetzt erst recht vor sich geht. Wenn die bestehenden Verordnnngen nach der Ansicht des Herrn Ge. lieimrat $J u c k e n a c k$ ausreichen, dann muß ich fragen: Warum haben wir eine Ersatzmitttelverordnung so mähsam aufgebaut? Ich bin der Meinung, daf die Ersatzmittelverordnungr nicht für Zeit und Ewigkeit aufgebaut war, aber auf der anderen Seite sage ich mir: Einreiben ist sehr schön, aber etwas anderes an seine Stelle setzen, ist schwierig und ich möchte, nachdem wir vor der vollendeten Tatsache stehen, bitten, daf das Nahrungsmittelgesetz mit der allergrößsten Beschleumigung ausgebant wird, damit wir für die nächste Zeit nicht einen Zustand bekommen, der blok zu Fehden in Gerichtssälen führt und zur Unsicherheit in der Nahrungsmittelindustrie. Wir haben gestern gesehen, wie es mit dem Kunsthonig geht. So wird es mit den anderen Sachen auch kommen. Es wird gesagt, wir haben die Preistreibereiverordnung. Es ist aber nicht sehr einfach, auf Grund davon vorzugehen, und die Lage bringt für die Fabrikabten auch eine groke Unsichetheit. Und wenn die Leute $\mathrm{z}$. $T$. auch freigesprocben worden sind, die unschuldig wareu, so bleibt doch etwas an ihnen hängen, und die anderen sagen, es muf doch etwas dran gewesen sein. lch komme auch von einem Begräb. nis erster Klasse, das wir in Baden gefeiert haben. Nachdem die Ersatzmittelverordnung für Lebensmittel aufgehoben war, blieb uns nichts weiter übrig, als daf wir die Verordnung uber fettlose Waschmittel anch aufhoben. Und es hat sich bereits gezeigt, dak das ebenfalls zu Sehwierigkeiten fuhrt. Wir haben bei fottlosen Waschmitteln nichts mehr in der Hand, als die Preistreibezeiverordnung. Ich bin fest uberzeugt, der Schwindel mit fettlosen Waschmitteln geht jetzt erst recht los. Ahnlich wird es bei den Nahrungsmitteln sein. Ich möchte von meinem Standpunkt als öfentlicher Chemiker hier offen aussprechen, daf ich es bedauere, daf die Ersatzmittelverordnung mit einem Schlage gefallen, und daß nicht automatisch das Nahrungsmittelgesetz an seine Stelle getreten ist.

Dr. Bouhon: Ich möchte anfragen, ob es nicht zweckmälig ist, in den $\$ 2$ oder 3 des Nahrungsmittelgesetzes die Bestimmung hereinzunehmen, daf die Entschädigung für die entnommenen Proben nicht in der Höhe des üblichen Kaufpreises sondern des Einkaufspreises zu zahlen ist.

Geheimrat Juckenack (unterbrechend): Kaufpreis ist der Einkaufspreis einschließlich der berechtigten Unkosten, nicht der Verkaufspreis.

Dr. Bouhon (fortahrend): Es wîrde sehr zu begruben seiu, wenn bei den Ausfuhrungsbestimmungen die Bestimmung über eine einheitlicho Durchlubrung der Lebensmittelïberwachung im ganzen Reiche getroffen würde. Bezüglich der Ersatzmittel bin ich auch anderer Ansicht wie Herr Prof. Beythien und ich wundere mich, daf gerade er als Sachse das sagt. Wir in Sachsen waren eigentlich selir stolz darauf. (Zuruf Beythion: Mit meiner Ausnahme!) Ich bin der Ansicht, dak eine Unmenge von alten Misständen wieder auftauchen wird, und die Ersatzmittel den Markt wieder tiberschwemmen werden.

Prof. Wirthle: Wir haben im vorigen Jahre in Eisenach beschlossen, nicht aufsondern abzubauen. Wir haben über die Wirkung der Uberwachung der Ersatzmittel gan 2 gemischte Erfabrungen gemacht. Vielfach ist man der Moinung: Jetzt bekommen wir das minderwertige Zeug wieder herein, wenn die Sache sang- und klanglos fällt, ohne daf ein El* satz dafür da ist. Ich fasse die Sache anders auf. Wenn auch die Nebenveroxdnungen gefallen sind, haben wir trotzdem noch oinen Anhaltspunkt, der nach meiner Ansicht vollständig für unsere Beurteilung ausreichend ist. Das sind unsere sogenannten Richtlinien. Daran müssen wir festhalten and ich bin fest uberzeagt, wir kommen durch. Wozu stehen wir in unserem Berufe drin und haben die langjährigen Erfahrungen gesammelt?

Prof. Rothenfufer: Die Ersatzmittelregelung ist tot. Es lebe die Ersatzmittelregelung. Wenn ich bis zum letzten Augenblick immer darauf hingewiesen habe, daf wir nicht ohne weiteres die Frsatzmittelregelungen von einem Tag auf den andern aufheben 
sollten, so war es die Überzeugung, daß dann Verhältnisse einreiß̉en wärden, die unhaltbar sind, und daßs wir damit ein Gebiet für gewisse stellungslose Schieber schaffen würden. Wenn jetzt dieses Vakuum eintritt, so ist die Gefahr selhr groß, und dieser Grund hat mich veranlafat, zu sagen, wir müssen abbanen, möglichst schnell abbauen, aber die Sache aus der Welt schaffen, ohne gleiclizeitig an ihre Stelle etwas anderes Besseres zu setzen, diesen Weg halte ich für sehr bedenklich. Nun ist von anderer Seite das Bedürfnis ausgesprochen worden, die Riehtlinien anfrecht zu erhalten. Gewif jst das wünschenswert. Aber Herr Geheimrat Juckenack hat gesagt, daßß davon keine Rede sein könne. Wir müssen also schauen, wio ,wir mit der Sache sonst fertig werden. Insbesondere werden sich auf dem Gebiete der übrigen Gegenstände des täglichen Bedarfs große Misstände zeigen. Es ist schon gestreift worden, was für Zustände bei uns in Sïddeutschland in bezug auf die fettfreien Waschmittel eingerissen sind. Es wird voraussichtlich im Bereich des Ersatzmittelmarktes ganz fürchterlich werden. Tch möchte den Antrag stellen, daßi wir uns über die Frage aussprechen, ob wir die Richtlinien stillschweigend als Grundlage für unsere Arbeiten und Beurteilungen anerkennen wollen, oder wio wir sonst während dieses Vakuums uns zu der Sache stellen.

Dr. Leonhard: Ich möchte kurz um eine Auskunft bitten. Nach dem Entwurf des teuen Nahrungsmittelgesetzes soll den Beamten der Untersuchungsanstalten weitgehende Befugnis zum Betreten der Ränme zur Erhebung von Proben gegeben werden. Ich möchte darauf aufmerksam machen, dą man dann Gefahr läuft, bei der Verhandlung als Sachverständiger abgelehnt zu werden, wenn man beim Vorverfahren als Hilfsbeamter der Staatsanwalischaft tätig gewesen ist. Ich möchte um Aufklärung über diesen Punkt bitten.

Geheimrat Juckenack: Wenn ich das Wort nehme, geschieht es deswegen, um die Debatte über die Ersatzmittelfrage abzuschneiden. Sie werden aus meinem gesamten Vortrage gesehen haben, daß3 ich mich bemüht habe, ganz sachlich alle möglichen Fragen der Lebens. mittelgesetzgebung auzuschneiden, ohne persönlich Stellang za nehmen. Nachdem die Ersatz. mittelfrage in die Dehatte geworfen worden ist, halte ich es tür meine Pflicht, einige Worte dazu zu sagen. lis ist hier gesagt worden, warum haben wir etwas aufgebaut, um es nachler wieder einzureisen. Wenn Sie der Begründnng der Reichsregierung gefolgt wären, hătten Sie gesehen, daf seinerzeit die Ersatzmittelverordnung geschaffen war, um gewissen Kriegs. schwindel energisch zu beseitigen. Fis ist nie daran gedacht worden, den Verkehr mit Ersatz mitteln jrgendwie dauernd einseitig zu regeln. Die Reichsregierung hat, bevor sie die Verordnung aufhob, sămtliche beteiligten Kreise gehört. Es waren in der entscheidenden Sitzung insgesamt ungefähr 100 Vertreter von Industrie, Handel und Verbrauchern, Konsumvereinen nsw. - von den Verbrauchern sind 25 Organisationen eingeladen gewesen - anwesend. Man war sich klar dariuber, daßs heute die Sachen, die seinerzeit als Unfug mit Recht bekämpft wurden, aus dem Verkehr verschwunden sind, und daf kein verständigex Menseh leute noch daran denkt, diesen Schund zu kaufen. Es wurde vom Kleinhandel selbst bestatigt, daf jene Sachen heute unverkafuflich sind, und auch von den Konsumvereinen wurde darauf hingewiesen. Meine Herren! Sagen wir es in unserem Kreise offen: Die Ersatzmittelverordnung war sohon seit einiger Zeit absolut undurchführbar. Was geschehen ist, das ist geschehen gegen die Rechtsgrundlagen, die vorhanden waren; sie war absolut nicht mehr durchführbar. Nachdem mun der Versailler Friede geschlossen war, trat ein derartiger sprunghafter Wechsel in den Preisen ein, daß es dem Kaufmann gar nicht mehr möglich war, zu kalkulieren, zumal er nicht wissen konnte, ob er dre Genehmigung bekam. - Non die zweite Frage: Kann man nicht von einer Festsetzung dex Preise absehen und die andern Sachen lassen? Da mufz jeh die Gegenfrage stellen: Können Sie es in einer Zeit, in des wir in der grö́sten Armut leben, und die Regierungen überlegen, wie sie noch einigermafien den fitat balancieren können, können Sie es da veranworten, nur für diese eine Frage die grofe Organisation der Ersatzmittelstellen - ich kenne Ersatzmittelstellen, die äber 60 Angestellte haben - mit ihren ungeheuren Kosten zn unterhalten? Lassen sich diese ungeheueren Kosten beute noch rechtfertigen? Nun betrachten Sie die Sache noch von einem anderen Standpunkte: Wenn Sie bedenken, dafs die ganze Gewerbepolizei in allen deutschen Ländern nur einen ganz kleinen Bruchteil der Sicherheitspolizei bilden kann, also nur einen gewissen Umfang haben kann, wenn Sie weiter überlegen, daḱ diese Gewerbepolizei nicht nur die Gewerbebetriebe, sondern auch das ganze Gebiet des Lebensmittelhandels überwachen soll, so werden Sie mir zustimmen, dals es unmöglich ist, daß̧ sie Kosten aufwendet fǘr die par Ersatzmittel, die, wenn Sie den gesamten Lebensmittelverkelir betrachten, doch nur einen gauz minimalen Bruchteil desselben bilden - daß sie also dafür Kosten anfwendet, die sie für die gesamte Nahrungsmittelkon. trolle nicht aufwenden kann. In Berlin sind mehr Beamte und Angestellte tätig gewesen zur Uberwachung des Verkehrs mit Eisatzmitteln als für den gesamten anderen Lebensmittelverkelr. Wie wollen Sie es rechtfertigen, für Saehen, die kein Mensch mehr kauft, eine derartige Organisation zu unterhalten? Für den Sachverständigen ist es natürlich bequem, feste Bestimmungen zu haben, aber man muß die Sache vom höheren Verwaltungsstandpunkte aus betrachten. Was das Publikum anbelangt, so war es interessant, daß geschlossen alle deutschen Konsum- 
vereine von der Grokeinkaufsgesellschaft herab bis zum Jetzten Konsumverein die sofortige Aufhebung verlangt haben, weil sie die Sache für unhaltbar und für gefährlich hielten. Nun die Richtlinien! Gewifs, sie haben heute nicht die formelle Bedeutung wie zur Zeit des Bestehens der Ersatzmittelverordnung. Sie bieten Ihnen hente aber doch die Möglichleit, im Zusammenhang mit der Verordnung gegen irreführende Bezeichnungen gegen Auswächso vorzugehen. Ich habe übrigens niemals ein Hehl daraus gemacht, daf es mir ferngelegen hat. irgendeiner Dienststelle, die bei der Ersatzmittelregelung beteiligt war, irgendeinen Vorwurf zu machen; der Grund, warum die Ersatzmittelverordnung versagt hat, liegt. lediglich darin, weil sie nicht durchfuhrbar war. Das lag an den wirtschaftlichen Verhaltnissen; wir wollen in der heutigen Zeit Gott danken, wenn es uns überhaupt möglich ist, die ganzen Mifistände allmählich so weit zu bekämpfen und einzudämmen, daß3 wir halbwegs exträgliche Verbältnisse bekommen. - Nun ist zum Schluß angeregt worden, die Überwachung überall einheitlich durchzuführen. Das halte ich für unmöglich. Sehen wir mal die Sache voin preußischen Stand" punkte an. Preußen erstreckt sich von der Ost- bis zur Westgrenze des Reiches und um. faß̧t Wirtschaftsgebiete aller Art. Man kann da alle möglichen Verlältnisse finden, wie es in kleineren Staaten nicht möglich ist. Die Verhältnisse sind in den einzelnen Gebieten Preufens ganz verschieden. Es jst etwas ganz anderes, ob ich in einem groben Industrieort kontrollieren lasse oder in Ostpreufen oder Pommern. Man muk die örtichen Verhältnisse yanz genau berucksichtigen und an Hand der Verbältnisse prüfen, in welehem Umfange die Kontrolle nötig ist. Die Zentralbehörde mufa auch zu den Anstalten das Vertrauen haben, dals sie ihre Polizeibehörde nach der Richtung beraten, daf3 solche Proben entnommen werden, mit denen wirklich weiter zu kommen ist. Wir erwarten, daß jeder bestrebt ist, daf sach gemäb kontrolliert wird, und ich muf3 sagen, mir hat jede Arbeit im Leben leid getan, die unfruchtbar war. Was hilft $ө$ s, wenn ich unbefriedigt meine Sachen weglege, wenn ich nicht auf dem Gebiete, wo ich verautwortlich bin, wirklich auch ersprieflich tätig bin. - Dann die Anfrage des Kollegen Leouhard wegen der Sachverständigentätigkeit: Ich habe mir die Sache so gedacht: Selbstverständlich dürfen die Nahrungsmittelchemiker nicht Hilfsbeamte der Stantsanwaltsehaft sein, denn dann können sie ohne weiteres vom Gericht abgelehnt werden. Was uns heute fehlt, ist das, daß die Sachverständigen befugt sein müssen, auch an den Revisionen teilzunehmen. Ich stehe grundsätzlich anf dem Standpunkte und fubre ibn in Preufán über. all durch, daß̧ der Sachverständige nie allein kontrollieren soll. Die Kontrolle wird durch die Polizei ausgeübt. Die Regel wird sein, daf der Sachverständige den Beamten richtig anlernt, aber formell ist dieser das Exekutivorgan und der Sachverständige ist nur der Berater. Einen anderen Weg halte ich, auch im Interesse des Ansehens der Sachverständigen, nicht für gangbar.

Geheimrat Abol: Sie haben die Liebenswürdigkeit gehabt, mir zu gestatten, dabs ich heute als Privatmann an ihren Verhandlungen teilnehme, nachdem ich so oft als behördlicher Ver treter ihnen beiwohnen durfte. Ich bin Ibnen dankbar dafür, um so mehr, als hier eine Angelegenheit erörtert wird, die mich schon lange beschaftigt hat. Bereits im Jahre 1907 habe ich einen Vortrag über die Reformbedürftigkeit unserer Nalurungsmittelgesetzgebung auf dem Hygienekongreßs in Berlin gehalten und 1910 einen solchen im Deutschen Verein für ôffentiliche Gesundheitspflege. Was hente von Herrn Geheimrat Juckenack vorgeschlagen worden ist, das möchte ich Wort für Wort nach meinen eigenen Erfahrungen als Hygieniker unterschreiben. Gestatten Sie, daf3 ich auf einige Punkte vielleicht näher eingehen darf, weil mir in ilınen das Wesentliche zu liegen scheint für eine wirklich zeitgemäße Ausgestaltung unserer Nahrungsmittelgesetzgebung. - Da scheint mir vor allen Dingen ein dringendes Bedürfnis zu bestehen, daf die ganzon Kontrollvorschriften im Nahrungsmittelgesetz anders gestaltet werden. Die Befugnisse, die im Nahrungsmittelgesetz der Polizei gegeben sind, reichen nicht entfernt aus. Die Beschränkung der Kontrolle auf die Verkaufsstelle allein, die Unmöglichkeit, in die Auf bewahrungsstellen einzudringen, ist immer wieder als störend empfunden worden. Es muf Einblick in die Verhältnisse des Nahrungsmittelbetriebes in dem Umfang gewonnen werden können, wie es zur Beurteilung der Sachlage nötig ist. Darüber wird kein Zweifel bestehen, ebensowenig wie darüber, daf nicht nur duch Polizeibeamte die Kontrolle vorzunehmen ist, sondern durch die Sachverständigen selbst, wo es irgendwie durchführbar ist. - Dabei möchte ich einen Punkt noch besunders hervorheben, den Herr Geheimrat Juckenack, jedenfalls nur aus Verseben, nicht erwähnt hat. Ein groß̉er Mangel besteht insofern, als die U̇ber wachung der von außserhalb zu uns kommenden Waren yanz im Argen liegt. Wir baben nur für einige wenige Dinge, die vom Ausland kommen, die Möglichkeit, sie zu kontrol. lieren. Es mus versucht werden, die Kontrolle über die Einfuhrwaren zu verschärfen. Mir will scheinen, als ob das heute sogar leichter durchführbar sei, als fraher, weil wir ja für eine grofe Zahl von Nahrungsmitteln noch eine Einfuhrbeschränkung liaben. Bei den jetzigen hohen Weltmarktpreisen ist die Gefahr noch gröser als sonst, da\} minderwertige Erzeugnisse ins Land kommen. - Dann scheint mir sehr wichtig ein Punkt, ühor den Sie sicher alle einer Meinung sind; daß nämlich in dem Gesetz nicht nur wie bísher die Möglichkeil des Erlasses ron $V$ orboten bestimmter Herstellungsverfahren usw. vorhanden sein soll, 
sondern daßz auch positive Vorschriften über die Herstellung und Beschaffenheit und die Bearteilung der wichtigsten Nahrungsmittel statthaft sein müssen. Gleichzeitig sollte die Kontrollbefugnis des Reichstags gegenüber solchen Vorschriften beseitigt werden. Es wird ihnen erinnerlich sein, daf̧ von der Möglichkeit, kaiserliche Verordnungen auf Grund des Nahrunsmittelgesetzes zu erlassen, im ganzen nur wenig Gebrauch gemacht worden ist, weil man eine kaiserliche Verordnung nicht der Kritik durch eine Zufallsmehrheit im Reichstag anssetzen wollte. Auch die jetzige Regierung wird sich nicht der Gefahr aussetzen, dafs eine von ibr erlassene Verordnung durch den Reichstag unwirksam gemacht wird. - Und nun noch eins, was Herr Geheimrat Jucke* n a ck schon erwähnt hat. Für eine Reiha von gewerblichen Betrieben ist eine Konzession nötig. Die Behörden können die Konzession wieder entziehen, wenn schwere Verstößre gegen die Interessen der Allgemeinheit in gesundheitlicher, wirtschaftlicher, sittlicher Hinsicht vorkommen. So kann nach der Gewerbeordnung $z$. B. sogar für den Schwimm-, Turn- und Tanzunterricht usw. die Konzession entzogen werden. Aber ein Nahrungsmittelfälscher kann seinen Betrieb immer wieder fortsetzen; wenn er seine Strafe bezahlt oder verbilist hat, fängt er von frischem an. Dabei stelt der Gewinn, der durch unlantere Manipulationen im Nahrungsmittelhandel erzielt wird, meist in keinem Verhältnis za der Strafe. Es müfte den Gerichten die Möglichkeit gegeben werden, zu bestimmen, dafs solchen Lebensmittelfabrikanten und Händlern, die dauernd den Volksinteressen entgegenhandeln, die Erlaubnis zur Fortsetzung ihres Betriebes entzogen wird. - Was die Bestimmungen des $\$ 10$ und 11 anbelangt, so kann ich mich dem anschliefen, was Herr $J$ uck enack ausgeführt hat. Vielleicht interessiert es Sie, wenn ich erwähne, daß die Paragraphen, die mich als Arzt besonders angehen, nämlich die Paragraphen 12-14, meiner Ansicht nach einer Umgestaltung nicht bedürfen. Während man bei der Schaffung des Gesetzes dieses in seinen wesentlichsten Beziehnngen als ein solches ansah, das gesundheitliche Schädigungen der Bevölkerung verhindern wollte, haben sich die Dinge inzwischen so verandert,- dafs man sagen kann: Das Nahrungsmittelgesetz ist heute im wesentlichen ein wirtschaftliches Gesetz. Gesundheitliche Schädigungen durch Nabrungsmittel sind glücklicher. weise selten geworden, und das Gesetz hat deshalb in dieser Beziehnng nicht molr dieselbe Bedeutung, die es bei seinem Erlaßs vor 41 Jahren besaß.

Dr. Hundeshagen: Wie stellen sich die Eisenbahnverwaltungen zu der gerade in der jetzigen Zeit, wichtigen lebensmittel - und gesundheitspolizeilichen Überwachung der Beförderungsmittel? Es scheint mir, daf diese Sache gegenwärtig noch sehr im Argen liegt. Es ist mir aus meiner eigenen Praxis beispielsweise ein Fall bekannt, dak eine grofe Sendung von Kakaobohnen, deren Wert hoch in die Hunderttausende ging, durch eine ganz unzweckmäßige Art der Beförderung und Unterbringung eine fast unberechenbare Einbuß̧e an Wert erfabren hat. Wie hat sich die Lebensmittelpolizei den Eisenbahnverwaltungen gegenüber zu verhallen, daß solche außerordentlich hohen Schädigungen des Volksvermögens vermieden werden?

Dr. Suhellbach; Ich wollte nur noch kurz oingehen auf die von verschiedenen Vorrednem gestreifte Frage der Waschmittel. Wer sicb damit befakt hat, der wird sagen, daß3 diese Waschmittelverordnung absolut nicht genügt hat. Der Schwindel auf dem Gebiet der Waschmittel war trotz der Verordnung riesengrof. - Nun möchte ich einen neuen Punkt in die Diskussion hineintragen. Es jst mir bei den Erörterungen des Kollegen Le on$l_{1}$ ard bezgl. der Sachverständigentätigkeit der Gedanke gekommen, ob nicht bei der Reorganisation der Rechtspflege die Frage angeregt werden sollte, ob es zweckmäßig ist, bei den Beratungen, zu denen sich die Gerichte nach der Verhandlung zurückziehen, auch die Sachverständigen zu hören und es würde ein grofser Erfolg sein, wenn auch bei der Nahrungsmittelgesetzgebung dieses ermöglicht werden könnte.

Dr. Baumann (schlecht zu verstehen) kommt auf $\$ 17 \mathrm{za}$ sprechen and bemängelt die Art der Verteilung der Strafgelder bei Verurteilungen.

Prof. Lendrich: Kollege Jucken ack hat die Grüde dargelegt, die zur Aufbebung der Ersatzlebensmittelverordnung gefuhrt haben. Ich teile diese Gründe durchaus. Auch wir haben gefunden, $\dot{d a}_{3}$ es in fruchtloses Arbeiten war. Aber die Ersatzlebensmittelverordnung hinterläbt noch einen Schatten. Was wird aus all den Sachen, die noch schweben und die gerichtlich anhängig gemacht worden sind auf Grund dieser Verordnung, die nun aufgehoben ist? Es ist ein eigentümliches Ding, sowohl für den Nahrungsmittelchemiker wie für den Richter, wenn sie gegen die Leute vorgehen sollen auf Grund einer Verordnung, die nicht mehr in Geltung ist. - Dann möchte ich bezgl. der $\$ 2$ und 3 des Nahrungsmittelgesetzes unsere Erfahrungen mitteilen in bezug auf die Erweiterung der Befugnisse der Lebensmittelüberwachung. In dieser Beziehung ist Hamburg vorbildlich vorgegangen, indem die Betriebsüberwachung eingefuhrt worden ist, die sogenannte Lex Heyl. Diese Mafnahme ist anferordentlich wirksam und segensreich, nieht nur in der Richtung hin, daf wir in der Lage sind, die versehiedensten Betriebe kennen $z u$ lernen, sondern anch insofern, als wir in der Lage sind, die Fabrikation selbst zu beeinflussen. Denn die Sachverständigen sind nicbt nur Revisionsbeamte. sondern auch Berater. Sie stehen den Indastriellen zur Seite, belehren sie, und nach dieser Richtung 
wird das auferordentlich angenehm empfunden und wir haben insofern keinerlei Schwierigkeiten. Wir haben diese Sache noch dahin erweitert, daß wir jetzt eine Auskunftsstelle geschaffen haben, wo täglich zwischen 9 und 10 Uhr ein Herr zur Verfügung steht, der den Verbrauchern, sowie auch der Industrie Auskünfte erteilt über irgend welche Fragen, die die Betreffenden besonders interessieren, z. B. Nahrungsmittelversorgung, Verfälschungen usw. Die von uns bisher gemachten Erfahrungen zeigen, daf auch dieser Weg beschritten werden kann und vielleicht auch von unseren Untersuehungsämtern weiter ausgebaut werden kann. Nur so können wir mit der Zeit anf die Bevölkerung und anf die Industriellen einwirken und unseren Ämtern viel Mühe und Arbeit ersparen. - Dann möchte ich noch einen Punkt erwähnen. Seitens der Handelskammern sind Anträge gestellt worden, dak man für die Überwachung der Betriebe gewerbliche Sachverständige heranzieben soll. Ich habe gegen diesen Vorschlag Bedenken geäufert, und zwar einmal Bedenken wirtsehaftlicher Natur, weil natîrlich nur solche Sachverständige gewählt werden können, die auch Erfahrungen in einschlägiger Beziehung haben, und es ist sehr miflich, wenn solche in die Betriebe der Konkurrenten hineinsehen. Zweitens halte ich es nicht für notwendig, denn die Sachverständigen, die diese Betriebe präfen sollen, besitzen ohne weiteres die Kenntnis, daß sie in der Lage sind, sachgemäß die Überwachung vorznnehmen. Es bedarf dazu nicht gewerblicher Sachverständiger.

Geheimrat Juckenack: Es wurde gesagt, dak wir bisher nach dem geltenden Recht nicht berechtigt seinen die Eisenbahnwagen und derartige Beförderungsmittel zu überwachen. Im preußischen Entwurf für Polizeiverordnungen ist jedoch auch vorgesehen, dafs die Kontrolle sich auch erstrecken soll auf die Beförderung vou Lebensmitteln, also kurz auf den gesamten Lebensmittelverkebr. Es ist natürlich notwendig, daß3 man die Nöglichkeit haben muß, die Lebensmittel auf dem gesamten Verkebr bis zum letzten Verbraucher kontrollieren zu können. - Die Anregung des Herrn Dr. Schellbach ist nicht notwendig. Wenn ein Gericht nach Schluf der Verhandlung uber eine Materie nicht klar ist, so braucht es nur die Urteilsverkündigung auszusetzen und hat dann 8 Tage Zeit, sich genan zu unterrichten. Woher es in der Zwischenzeit seine Kenntnisse genommen hat, das ist seine Sache. - Hern Dr. Baumann möchte ich darauf hinweisen, dafs gerade die von ihm angeschnittene Frage sehr eingehend in einem früheren Vortrage von mir behandelt worden ist, der auch in unserer Zeitschrift abgedruckt ist. Es muß jedesmal bei der Geidstrafe die Feststellung des Gerichts geprüt werden, warum der betreffende Täter verurteilt worden ist. Wenn es heifst, er ist verurteilt wegen Herstellung un d Verkaufs verfälschter Nahrungsmittel und die Tatorte decken sich nicht, so muf die Geldstrafe geteilt werden. Ich will hier nicht näher darauf eingehen, sondern müchte nur auf meinen damaligen Vortrag verweis $n$. Darin ist die Sache eingehend behandelt. - Was die Frage betriff, inwieweit noch Verurteilungen auf Grund der aufgehobenen Verordnung zu erfolgen haben, so möchte ich darauf hinweisen, daß wir an sämtliche Ersatzmittelstellen einen Runderlaß wesendlichen geregelt ist. Wir haben darin gesagt, daßs die Ersatzmittelverordnung aufgehoben ist und nichts mehr veranlafit werden soll. Im ubrigen müssen natürlich die Sachen, die beim Gericht anhängig gemacht worden sind, durchgefuhrt werden, weil ja für die Gerichte dazu die Pflicht besteht. I ch bin im übrigen selbst der Ansicht, daß man in den letzten 14 Tagen keine Sache anhängig machen sollte. Natürlich wenn die Tat vor dem 1. Oktober liegt, muf3 eingeschritten werden. - Zum Schlußs berührte Kollege Lendrich eine sehr interessante Frage, die auch in anderen Orten schon akut geworden ist, nämlich die Frage der amtlichen Auskunftserteilung. Die Frage hat im Laufe der letzten 20 Jabre wiederholt Anlás zu Erörterungen gegeben. Es ist eine schwierige Frage. Die maßsgebliche Auslegung der Gesetze steht ausschlieblich den Gerichten zu, und es ist immer bedenklich, mündliche Auskánfte zu erteilen, weil späterhin nie genau der Wortlaut der Auskunft restgestellt werden kann. Im Ministerium geben wir derartige Auskünfte nicht. Wir pflegen zu sagen, daß die Auslegung der Gesetze Aufgabe der Gerichte sei, und daßs wir im übrigen der Ansicht seien, daßs, wenn die Sache so und so gehandhabt werde, kaum Bedenken bestehen wärden. Man muf sich da immer sehr vorsichtig äußern. Wesentlich ist auch, dafi derartige Auskünfte schriftlich gègeben werden, denn vielfach werden vom Publikum die Auskünfte unrichtig aufgefaft. So hatten wir einen Fall, wo wir vor zwei Jahren eine schriftliche Auskunft erteilt haiten. Wir haben das Konzept vorlegen können und konnten nachweisen, daf ich die Auskunft gerade im umgekehrten Sinne erteilt hatte, wie der Betreffende behauptete. Wir erteilen deshalb stets die Auskünfte der Vorsicht halber mit der eben erwähnten Einschränkung.

Prof. Wirthle: Tch möchte nur hervorheben, dafs die Erfolge bei unserer Untersuchungsanstalt nicht zum geringsten darauf beruhen, daßs die Beamten bei der äußeren Kontrolle nicht nur Polizeibeamte spielen, sondern den Herstellern, den Kadfleuten und den Behörden beratend zur Seite stehen und dadurch das Vertraten aller dieser Kreise gewonnen haben. Bezgl. der Erweiterung des \& 2 unseres Nahrungsmittelgesetzes möchte ich sagen, daf wir in Bayern dureh nnsere Polizeivorsehriften seit mindestens 30 Jahren befugt sind, das zu tun, was in der nouen Fassung des Nahrungsmittelgesetzes vorgeschlagen wird, und dak 
wir befugt sind, sowohl in die Herstellungs- als auch in die Aufbewahrungs- and Verkaufsräume einzudringen und da die Kontrolle vorzunehmen und Proben zu entnehmen. Nun sind wir bei diesen Kontrollen keineswegs Polizei, anch nicht Hilfsbeamtè der Staatsanwaltsehaft, sondern wir sind nach unserer Verordnung lediglich Sachverständige der betreffenden Verwaltangsheamten, selbst dann, wenn, was sehr băfig der Fall ist, die Kontrolle von uns allein ansgeubt wird. Ich habe schon wiederholt die Erfahrung machen müssen, dak, wenn ich in der einen oder anderen Studt den. Bürgermeister mitnehme, der gleichzeitig ein Geschäft hat, dann ist der Teufel los, und mit Recht. Die Lente erblicken in ihm einen Konkurrenten und sagen: lhnen, Herr Dr. sag ichs, dem sag ichs nicht. Da muß ich den Bürgermeister bitten, das Lokal zu verlassen. Fr ist der zustündige Verwaltungsbeamte, und der Sachverständige muf ihn bitten, einen Augenblick vor die Türe hinauszugehen! Das ist natüriich ein unhalt. barer 7instand.

Dr. Stadlinger: Ich möchte nur auf das Bezug nehmen, was Herr Geheimrat $\mathrm{A}$ bel erwähnt hat bezgl. der Entziehung der Konzession im Falle wiederholter schwerer Verletzungen der Bestimmungen. Nach Abs. 3 im Artikel 151 der Verfassung des Doutsehen Reiches vom 11. Angust 1919 heißst es: Die Freiheit des Handels and Gewerbes wird nach Mafogabe der Reichsgesetze gewährleistet". Es kann sich einer darauf berufen. daf er erklärt: „Ich kann zufolge Reichsverfassung ohne weiteres wieder arbeiten!" Deshalb wärde sich empfehlen, im Nahrungsmittelgesetz durch eine Sonderbestimmung darnuf hinznweisen, daf alle nnzuverlässigen Personen, die direkt oder indirekt an dex Lebensmittelprodaktion und am Lebens. mittelhandel teilnehmen, auszuschliefien sind. Es muf anch verhütet werden, daßs ein soleher "Maulwurf" nicht in Form einer G. m. b. H. oder sonstwie unter Decknamen sein nnsauberes Hand werk weiter betreiben kann. Ich möchte deshalb besonders auf den Artikel 151 der Reichsver. fassung hingewiesen haben. Die Ausführungen des Kollegen Schellbach, daf sich die Waschmittelverordnung vom 11. Mai 1918 nicht bewährt habe, mobhte ich dahin richtig stellen, daf sich die Waschmittelverordnung als solehe wohl gut bewährt hat, aber dies nicht in ihren Answirkungen zeigen konnte, weil die Kontrolle der fettloson Waschmittel nicht ansgiebig genug war.

Dr. Baumann: Was Herr Geheimrat Juckenack als Antwort anf meine Anfrage gesagt hat, war mir anch bekannt. Aber meine Wïnsche gehen weiter. Wenn wir Falschungen in anderen Bezirken nachweisen, möchten wir gerne die ga $\mathrm{nzen}$ Strafgelder haben. Wenn wir die Arbeit leisten, so möehten wir auch das Geld haben. Ich glaube, dieser Wimsch ist gar nicht so unberechtigt.

Prof. Behre: Tch kann mich zur Frage der Kontrolle kurz fassen, nachdem ich, wie Sie wissen, in einer Veröffentlichung in der Chemikerzeitung meine Ansicht in dieser Frage mitgeteilt habe. Es muß, um mich kurz zu fassen, eine. Verschiebung des Schwergewichts der Tätigkeit ans dem Laboratorium in die Aufenkontrolle, in die Fabrikkontrolle, stattfinden. Mich interessiert noch eine andere Frage, nnd in dieser Beziehung möchte ich Herrn Geheim: rat Juckenack fragen, wie stellt sich die preubische Regierung und wie stellen Sie sich $2 u$ der Frnge der Einheitlichkeit der ganzen Kontrolle im Reich? Wir wissen, daß die Ein. heitlichkeit noch sehr schlecht ist. Was die Frage der Verteilung der Proben in den einzelnen Landern angeht, so stehe ich auf dem Standpunkt, dab das nicht gleichmäig gehandhabt werden kann. Aber eine gewisse Gleichmäßsigkeit im ganzen Reiche muß doch erzielt werden können. Wie denkt sich nun die prenßische Regierung, wie denkt sich das Reich die Uberwachung in den einzelnen Ländern, damit eine gewisse Einheitlichleit der Lebensmittelüberwachung ausgeubt wird. Das liegt nicht nur in unserem Interesse, sondern auch im Interesse des redlichen Handels.

Prof. Haupt: Ich wollte auf die Schwierigkeiten hinweisen, die den jüngeren Herren bei den Kontrollen in der Jndustrie entgegenstehen. Wenn man jahrelang in dieser Hinsicht tătig ist, dann weiß man, daß es nicht von honte auf morgen möglich ist; solche Betriebe za übersehen. Es würde sich empfehlen, wenn bei der Ausbildung und bei Fortbildungskursen auf diesen Punkt ganz besonderer Wert gelegt würde. Fis wäre sehr zweckmäkig, . wenn die Kollegen, die neuerdings mit solchen Arbeiten betrant werden, vorber Ge legenheit hätten, unter sachkundiger Führnng solche Betriebe zu besichtigen. Denn man steht: zunächst, wenn man zum ersten Male in einen Betrieb kommt und dort kontrollieren soll, manchmal dümmer da, als der Werkmeister, trotz allen theoretischen Kenntnissen, die man hat. Bezüglich der Transportmittel kann ieh dasselbe bestätigen, was hier schon erwähnt worden ist, and ich meine, wenn die Reichsregierung nach dieser Richtnng hin Abhilfe schaffen könnte, so wîrde das nur im allgemeinen Interesse liegen.

Dr. Sehellbach: Auf die Antwort des Herrn Geheimrat Juckenack war ich gefant. Das war mir bekannt. Es ist das aber unter Umständen nur eine rein theoretische Erörterung für das Gericht. Demn wenn zu einem Termiue 12 Sachverständige aus allen möglichen Städten, sogar ans Süddeutsehland, hinzugezogen worden sind, wird sich das Gericht wohl äberlegen mitssen, ob es sich durch eine Vertagung die hohen Kosten aufladen soll. 
Prof. Lendrich: Die Bedenken, die Kollege $J$ uckenack gegen die Anskunftserteilung hat, kann ich nicht teilen. Es kommt selbstverständlich darauf an, daß der Sachverständige, der diese Auskunft gibt, sie auch so vorsichtig und so umgrenzt gibt, daß $\mathrm{ihm}$ daraus nicht irgendwie ein Strick gedreht werden kann. Außerdem wird die Auskunft. auch nur mündlich erteilt, so daf schriftliche Unterlagen nicht bestehen. Dann behandeln diese Auskünfte auch meistens nur gewerbliche Fragen oder Gesetzesauslegungen. Und letzten Endes haben auch die Verbraucher und die Indnstriellen ein Recht daranf, sich irgendwo Auskunft holen zu können. Denn wer soll sie letzten Endes geben? Ich habe oft die Erfahrung gemacht, dak die Gerichte diese Lente einfach an uns verwiesen, weil das Gericht selbst nicht in der Lage war, eine Auskunft zu erteilen.

Dr. Mezger: Bei uns in Stattgart uben die Polizeiorgane die ambulante Lebensmittel. uberwachung aus. Bezüglich der Bestrafung wïrde ich es für zweckmäfig halten, wenn die be. treffenden Geschäftslente, die wegen irgend eines geringen Verstoßes angezeigt werden, nicht. gleich in hohe Strafe genommen werden, sondern ich wïrde dafür sein, da.f man sje zunäehst. nur verwarnt. - Dann kommt es auch vor, daf eine ganze Reihe von Kontrollen ergebnislos verlaufen, und der Täter nicht ermittelt werden kann. Es wäre in solchen Fällen angebracht, wenn die Sache, die von vornherein anssichtslos ist, nicht weiter verfolgt und das Amt nicht mit Akten belastet wïrde. Es könnte höchstens die Frage entstehen, wer bezahlt die Kosten der Unter. suchung. In diesen Fällen sind wir in der Praxis daza übergegangen, den betreffenden auswärtigen Beamten daranf hinzuweisen, daf an dem und dem Tage eine Anzeige erfolgt sei und dafs wir die Sache ad acta gelegt haben. Ob das gesetzlich zulässig ist, möchte ich bezweifeln, aber wir haben uns damit behelfen müssen, um die Akten nicht ins Ungeheure anwachsen zu lassen. - Die Frage der Auskunftsstellen ist anch schon an uns herangetreten, und ich mußs sagen, daß es tatsulchlich notwendig ist, daß man dem Gewerbetreibenden mit Rat znr Seite steht und ihm nicht nur sagt, was verboten ist, sondern auch was erlanht ist, wie die Zusammensetzang sein muß usw. Es ist auch meine Ansicht, daf man die belehrende Tatig. keit der Nahrungsmittelehemiker mehr in den Vordergrund ritcken muf. Es kann doch keine Befriedigung bieten, jeden Gewerbetreibenden vor den Kadi zn schleppen und in dem einen Fall obznsiegen, in dem anderen Falle zu unterliegen, je nachdem der Betreffende in der Lage ist, einen Sachverständigen aufzutreiben und zu bezahlen oder nicht. Die Strafe soll nur ein Mittel zum Zweck sein, um Bessorung zu schaffen. In erster Linie sollte man mildere Maßnahmen anwenden, und wenn im Nahrungsmittelgesetz die Möglichkeit gegeben wäre, eine Verwarnung vorzasehen, so würde ich das für sebr nützlich halten. - Was Herr Hundeshagen gesagt hat bezgl. des Verderbens von Nahrungsmitteln auf der Eisenbahn kann ich nur bestätigen. Wir haben in Württemberg eine Unmenge von Obst verderben sehen als Folge nnzweckmäßiger Verpaekung im Waggon, obne daß der Waggon vorher gereinigt war usw.

Dr. Willeke: Ich möchte der Anregung zustimmen, die Herr Geheimrat A bel hier gemacht hat bezgl. der Uberwachung der Einfuhrwaren. Ferner möchte ich anch der $A n$ regung des Kollegen Behre zustimmen, eine Vereinheitlichung der Kontrolle im Reiche an. zustreben. Wir hahen in Hessen-Nassan in dieser Beziehnng eine Buntscheckigkeit, die kaum glaublich ist. Es wäre ferner auch zu erwägen, ob nicht die Zollbehörden ersucht werden sollen, die amtlichen Anstalten mehr zu bevorzagen, um gerade in der Richtung hin ein Zusammenarbeiten in der Lebensmittelüberwachnng zu ermöglichen. Vielfach gehen die Zollbehörden selbständig vor, lassen die Sache untersuchen und đie Lebensmittelüberwachung bekommt erst. post festum Kenntnis von der Sache. Das ist auch ein Mifistand.

Dr. Theopold: Wenn man am Schlusse des Jahres das Facit aus seiner Tätigkeit zieht, ist man in vielen Fallen recht unbefriedigt. Man hat vielfach Tuebensmittel beanstandet. und findet dasselbe Erzengnis noch in Dutzenden von Geschäten vor, ohne dab die Möglich keit gegeben ist. die beanstandeten Lebensmittel einzuziehen. Die Einziehnng bezieht sich nur auf die beanstandeten Waren. Es müBte im neven Nahrungsmittelgesetz ein Weg gefunden werden, alle als verfalscht erkannten Lebensmittel aus dem Handel zu ziehen.

Prof. Wirthle: Eine einheitliche Ausfïhrung der Lebensmittelüberwachung ist nicht möglich. Nelimen wir z. B. unsere bayerischen Verhältnisse. Die Leute sind 30 Jahre an Kontrolle gewöhnt. Man darf da keine schematischen Vorsehriften erlassen.

Der Vorsitzende: Teh frage den Berichterstatter, ob or auf die letrten Anregungen noch etwas zu erwidern hat.

Geheimrat Juckenack: Zunichst die Waschmittelverordnung: Die Sache hat von vorn. herein daran gekrankt, daf es tatsächlich keine Ersatzmittel für Seife und Soda und Pottasche gibt, und wir Sachen genehmigen mußten, die kein Ersatz waren. - Was die Einheitlichkeit der Kontrolle betrifft, so beraten wir zur Zeit darüber einheitliche Grundsätze für die Durch. führung der Weinkontrolle aufzustellen, und es wird vielleicht auch möglich sein, Richtlinien zu beraten, wenn wir das Nahrungsmittelgesetz ändern. Es kann sich dabei aber immer nur darum handeln, Richtlinien zi vereinbaren. - Was die Anregung des Kollegen 
Ha u pt, über Fabrikkontrolle angeht, so ist der beste Weg, jüngere Kollegen anzulernen, der, daf 3 man sie bei Kontrollen mitnimmt. Allerdings ist es zweckmäfig, wenn man schon in den Hoehschulen anfängt, im Kolleg und bei den Besichtigungen darauf hinzuweisen. - Herm Sebellbach möchte ich sagen: Man maeht das einfach so, man bringt das Verfahren zum Abschluj 3 und setzt nar die Urteilsverkïndung aus. - Mit Bezug auf die Ausfährungen des Herrn Lendrich weise ich darauf hin, daf ich nicht gegen die Einrichtung von Auskunftsstellen sprechen sondern nur auf die Bedenken hinweisen wollte. Man muf jedentalls sehr vorsichtig sein. - Dann die Auslandswarenuberwachung: Der Gedanke ist außerordentlich wertvoll. Was mir jedoch im Augenblick noeh nicht klar ist, ist die Frage, wie die Kosten aufgebracht werden sollen. Man kann so etwas leicht machen bei der allgemeinen Verstantlichung, denn dann schützt man die gesamten Bewohner des Staates. Violleicht kann man die Kosten auf die Einführenden abwälzen, so wie wir es bei der Auslandsfleischkontrolle haben. Die Sache scheint mir jedenfalls außerordentlich wichtig zu sein. - Dann wegen der Verwarnungen. Da scheint eine ganz falsche Vorstellung zu herrschen. Eine Verwarnung ist grundsätzlich keine Strafe. Eine Warnung kann selbstverständlich jeder aussprechen, der nicht Staatsanwalt ist, weil nur diese Kategorie von Beamten verpflichtet ist, die Verfehlungen weiterzuverfolgen. - $\mathrm{Zu}$ den Ausführungen dès Herrn Wirthle hinsichtlich der besonderen landesrechtlichen Bestimmungen in Bayern möchte ich darauf hin weisen, daß ich auch in meinem Vortrage bemerkt habe, daß unbedingt $\$ 4$ Abs. 2 des Nahrungsmittelgesetzes bestehen bleiben muk, wonach die Landesbehörden befugt sind, weitergehende rechtliche $\mathrm{Be}$ stimmungen zu erlassen.

Der Vorsitzende: Dann hätten wir hiermit den ersten Punkt des Referates in einer genügenden Aussprache behandelt. Ehe wir nun zur Debatte über den zweiten Teil schreiten, möchte ich Herrn Kollegen $\mathrm{K}_{0}$ ber bitten, das uns zugesagte Referat zu erstatten.

\section{Vorschläge über die Finanzierung kommnnaler Unter- suchungsanstalten. \\ Von}

Kober-Trier.

Die diesjährige Etatsaufstellung für die Nahrungsmittel-Untersuchungsämter hat wohl jedem damit Beauftragten Schwierigkeiten gemacht. Der größte Posten in der Etatsaufstellung heißt „Zu deckendẹ́r Fehlbetrag". Ich schlug unserem Vorsitzenden vor, über die Deckung dieser Fehlbeträge zu beraten, und wurde daraufhin zum Referenten dafür bestimmt.

Sie wissen alle, meine Herren, wie schwierig die finanzielle Lage der kommunalen Untersuchungsämter geworden ist. Die Ausgaben sind gegen den letzten Friedensetat um das $4 \frac{1}{z^{-}}$bis 6 -fache gestiegen. Die Einnahmen können damit längst nicht mehr Schritt halten. Nachfolgenden Ausführungen liegen die Verhältnisse eines städtischen Untersuchungsamtes im Weinbaugebiete zugrunde, dem eine Anzahl Landkreise durch Verträge angeschlossen sind. Das Amt hat für die Landkreise eine festgelegte Mindestzahl von Untersuchungen auszuführen und zwar auf je 400 Einwohner der Landbevölkerung und je 200 Einwohner der Stadtbevölkerung eine Probe. Von den Proben sollen $4 \%$ auf Weinuntersuchungen entfallen. Dies macht für einen Amtsbezirk mit meist ländlicher Bevölkerung von 450000 Einwohnern etwa 1300 Proben im Jahr aus. Die Polizeiverwaltungen sind verpflichtet, diese Mindestzahl an Proben einzusenden. Sind nun die Gebührensätze niedrig, so wird die einzelne Polizeiverwaltung nicht allzu ängstlich abwägen, ob diese oder jene Probe auch wirklich zur Beanstandung führt, und wird sie, die vorgesehene Mindestzahl überschreitend, 